www.jmscr.igmpublication.org

Impact Factor (SJIF): 6.379

Index Copernicus Value: 71.58

ISSN (e)-2347-176x ISSN (p) 2455-0450

crossref DOI: _https://dx.doi.org/10.18535/jmscr/v6i4.96

Journal Of Medical Science And Clinical Research

IGM Publication

An official Publication of IGM Publication

\title{
Vestibular Evoked Myogenic Potentials in Benign Paroxysmal Positional Vertigo: Will it be a potential diagnostic tool?
}

\author{
Authors \\ Anish Bava Salim*, Dali Prabhakaran, Shyja \\ Kannur Medical College, Kannur, Kerala \\ *Corresponding Author \\ Dr Anish Bava Salim \\ Associate Professor, Kannur Medical College, Kannur, Kerala \\ Email:dr.anishbhava@gmail.com
}

\begin{abstract}
Objectives: To investigate the incidence of VEMP abnormalities in patients suffering from BPPV.

Methods: 30 consecutive patients referred to our vertigo clinic were selected as per the inclusion and exclusion criteria and evaluated as per the standard vertigo protocol. VEMP was performed on patients with BPPV and results compared to other parameters by detailed statistical analysis.

Results: We observed that VEMP was not a helpful tool in diagnosing BPPV. There was no correlation between VEMP changes and BPPV, but VEMP is an additional tool to the currently available tests for peripheral vertigo.

Conclusions: According to our study VEMP was not a useful test in diagnosing BPPV.

Keywords: Vestibular Evoked Myogenic Potentials, Benign Paroxysmal Positional Vertigo, sternocleidomastoid muscle, Rhomberg's test, Dix' Hallpike's test.
\end{abstract}

\section{Introduction}

Vestibular evoked myogenic potentials (VEMPs) are electromyographic (EMG) responses to loud auditory stimuli that can be recorded in the sternocleidomastoid (SCM) muscle during tonic contraction. Bickford et al. ${ }^{[1]}$ is the first to record these EMG potentials and Murofushi et al. [2] proved that VEMP are produced by a reflex arc that involves the vestibular saccule, the inferior vestibular nerve, and the SCM muscle. Benign paroxysmal positional vertigo (BPPV) is the most common disorder of the peripheral vestibular system characterized by episodes of vertigo associated with head movements. In explaining the pathophysiology of BPPV, the concept of a degenerative process that affects the macula of the utricle causing detachment of otoliths has gained popular support ${ }^{[3]}$. However, Akkuzu et $\mathrm{al}^{[4]}$. suggested that this degenerative process might also affect the macula of the saccule. In addition, Gacek $^{[5]}$ reported ganglion cell degeneration of the saccular nerve in a post-mortem examination of BPPV patients. Further, saccular degenerative changes can be detected by testing of VEMP, and this type of testing could be valuable for assessing BPPV patients.

So far, many authors have reported some pathognomonic findings or results for specific vestibular disorders like Meniere's disease and superior semicircular canal dehiscence ${ }^{[6]}$. In this 
study, we search for any VEMP changes specific for Benign Paroxysmal Positional Vertigo.

\section{Patients and Methods}

The study was conducted 30 consecutive BPPV patients referred to the Vertigo Clinic at St. John's Medical College.

Patients with positional vertigo and /or positional nystagmus and with the age group of 30-70 years were included in the study.

Further, patients on vestibular sedatives and drugs causing vertigo, Moribund/sick patients who cannot follow commands and patients with Diabetes mellitus/Hypertension/cardiac diseases/ cervical spondylosis were excluded from the study.

Then the patient's detailed history was collected and a neuro-otological examination was performed. Further, the patients were subjected to blood tests (Hematology \& biochemistry for sugar control assessment), X-ray of the Cervical spine (AP and lateral), carotid and vertebral artery Doppler Study, Electronystagmography (ENG) and VEMP.

Further, the patients for other clinical tests like

$\checkmark$ Rhomberg's test- to check whether the patient sways or falls when the eyes are closed.

$\checkmark$ Sharpened Rhomberg's test - to check the patients balance ability.

$\checkmark$ Dix' Hallpike's test- to detect vertigo and clinician observation of nystagmus

$\checkmark$ Tandem Walking- to detect truncal ataxia.

\section{Procedure for VEMP}

VEMP is done in our hospital using Eclipse Platform. The electrode used on the forehead is called ground electrode. Two electrodes are used on either side of mastoid. Two electrodes called vertex electrodes are placed on both SCM. When stimulus begins patient must raise his/her head to contract SCM muscle. Turning the head slightly to the opposite side helps to increase the muscle tonus. This position is held during the whole VEMP recording. Optimum results are obtained when patient is trained to maintain contraction of SCM during stimulation. The VEMP response is demonstrated by reproducible wave deflections with latencies around 13-23 milliseconds. VEMP morphologies and exact latencies are stimulus and patient dependent. It is common to run 2-4 recordings in the same sitting to ensure good reproducibility with only 100-200 stimulus presentation per recording. It takes approximately half - one minute per side. The VEMP result is evaluated by 2-4 reproducible wave form with deflection at approximately 13 and 23 milliseconds. The first positive peak is called P1 or P13 and negative peak N1 or N23. From each composite average, $\mathrm{P} 1$ and $\mathrm{N} 1$ peak latencies and P1-N1 amplitude are measured. Amplitude and latency asymmetries between right and left side are compared using the following asymmetry ratio calculation. Right P1-N1 amplitude -Left P1N1 amplitude/Right P1-N1 amplitude +Left P1- N1 amplitude. The ratio is calculated using stimulus intensities greater than $90 \mathrm{db}$ to ensure a suprathreshold stimulus. Data entry was done for all the parameters tested.

\section{Statistical Methods}

Descriptive statistics reported using numbers and percentage for the categorical variable and Chisquare test or Fisher's exact test was done to test the association between the VEMP normal and VEMP abnormal with demographical, clinical and laboratory variables. The analysis was done using the statistical software STATA/IC 12.1 and $\mathrm{p}<0.05$ is considered as significant.

\section{Results}

Out of 30 patients in the study group, Male and females were found to be almost equal in number with a slight female preponderance. The mean age group was 50 years. The youngest patient was 32 years and the oldest 68 years.

In the present study, most of the patients had giddiness lasting 5-10 seconds while few had giddiness lasting upto two mins with lightheadedness lasting upto 10 minutes. Further, one patient had giddiness which lasted an hour. 
Regarding the severity of $48 \%$ had experienced mild giddiness, $41 \%$ had severe giddiness and the rest had moderate giddiness.

Further, in the present study $50 \%$ of the patients had tinnitus as an associated feature, of which $62 \%$ had continuous giddiness and the rest had intermittent tinnitus.

The results of the Rhomberg's test reveal that more than $90 \%$ of patients was negative and the rest of the patients had a positive rhomberg's, but almost all of those positive patients had presented during an acute event.

Meanwhile, Dix hallpike diagnostic test for vertigo was positive in $90 \%$ of patients, the remaining 3 had no nystagmus on Dix hallpike testing but subjective giddiness during the test.
Based on Puretone audiometry (PTA) 55\% of the patients had normal PTA while $20 \%$ had mild SNHL and $20 \%$ mild CHL on pure tone audiometry.

Further, based on the ENG analysis $80 \%$ of the patients were normal and the rest of them elicited abnormal response with one displayed defect in visual fixation and saccadic movements.

Finally in our study VEMP was normal in $90 \%$ of patients. Three had abnormal VEMP findings with asymmetry in $\mathrm{p} 1$ latency and $\mathrm{n} 1$.There was no consistent changes in VEMP.

\section{Comparison of VEMP with clinical findings}

In our study, we found that two patients having abnormal VEMP findings did not have tinnitus as a symptom and the results were found to statistically non significant (Table 1).

Table 1: Comparison of VEMP with symptomatology (Tinnitus)

\begin{tabular}{|c|c|c|c|}
\hline & $\begin{array}{c}\text { VEMP } \\
\text { Normal }\end{array}$ & $\begin{array}{c}\text { VEMP } \\
\text { Abnormal }\end{array}$ & p value \\
\hline Tinnitus Absent & $12(46.15)$ & $2(66.67)$ & 0.598 \\
Present & $14(53.85)$ & $1(33.33)$ & \\
\hline
\end{tabular}

Further, all the patients who tested abnormal for VEMP had negative Rhomberg's. Sharpened normal in our study and was statistically non Rhomberg's and Tandem walking tests were

Table 2: Comparison of VEMP with clinical tests

\begin{tabular}{|l|l|l|l|}
\hline Clinical tests & $\begin{array}{l}\text { VEMP } \\
\text { normal }\end{array}$ & $\begin{array}{l}\text { VEMP } \\
\text { Abnormal }\end{array}$ & p value \\
\hline $\begin{array}{l}\text { Rhomberg } \\
\text { Negative } \\
\text { Positive }\end{array}$ & $\begin{array}{l}24(92.31) \\
2(7.69)\end{array}$ & $\begin{array}{l}3(100.00) \\
0(0.00)\end{array}$ & 1.000 \\
\hline $\begin{array}{l}\text { Sharpened Rhomberg } \\
\text { Negative }\end{array}$ & $20(76.92)$ & $\begin{array}{l}3(100.00) \\
\text { Positive }\end{array}$ & 1.000 \\
\hline $\begin{array}{l}\text { Tandem walking } \\
\text { Normal } \\
\text { Abnormal }\end{array}$ & $21(80.00)$ & \\
\hline
\end{tabular}

Furthermore, of the patients who had a normal ENG, one had abnormal VEMP and of the three who had abnormal ENG, one had abnormal significant (Table 2).

Table 3: Comparison of VEMP with Electronystagmogram

\begin{tabular}{|l|c|c|c|}
\hline Clinical Test & VEMP normal & VEMP Abnormal & p value \\
\hline ENG & & & \\
Normal & $22(84.62)$ & $1(33.33)$ & 0.062 \\
Abnormal & $3(11.54)$ & $1(33.33)$ & \\
Not done & $1(3.85)$ & $0(0.00)$ & \\
\hline
\end{tabular}




\section{Discussion}

Vestibular-dependent myogenic responses to intense sound were first described by Bickford et al ${ }^{[1]}$. VEMP was widely used since the work of Halmagyi and Colebatch ${ }^{[7]}$. The gold standard vestibular function test is Electronystagmography $(\mathrm{ENG})^{[8]}$. The caloric test induces vertigo and assesses only the horizontal semicircular canal function ${ }^{[9]}$. Compared to the ENG, VEMP testing is easier to perform, less complicated for interpretation, induces less dizziness or nausea, and is more tolerable to patients ${ }^{[10]}$. The pathway of VEMP circuit starts with the sound stimulating the saccule, which activates the inferior vestibular nerve, lateral vestibular nucleus, 11th nerve nucleus, and then ending with the sternocleidomastoid muscle (mostly ipsilaterally) through the vestibulospinal tract ${ }^{[11]}$. The sternocleidomastoid muscle has more homogenous responses than other muscles ${ }^{[12-14]}$. Gacek ${ }^{[15]}$ reported loss of vestibular ganglion cells in the inferior vestibular nerve, ganglion degeneration in the saccular nerve in the temporal bones of the BPPV patients. Shucknedt ${ }^{[16]}$ proposed the theory of "cupulolithiasis" and Hall [17] proposed the concept of "canalolithiasis", explain the pathogenesis of BPPV. Currently, Epley's canalith theory explains most of the features of BPPV ${ }^{[18}$, 19]. The Dix-Hallpike positional test is the gold standard for diagnosing posterior canal BPPV. However, patients experiencing lower back, cervical spinal problems or for the elderly, rotation and extension of the neck during the positioning may require a caution ${ }^{[20]}$.VEMP as a diagnostic tool has been proposed for BPPV in such patients. There has been a recent interest in tapping the potential of this investigation in various vestibular disorders. So in this backdrop, present study was to report VEMP findings in BPPV and hopefully to identify some clinical applications of this test in this patient group.

The study included patients who were referred to the vertigo clinic from ENT as well as neurology, orthopaedics, cardiology etc. We included 30 patients (of more than 200 total vertigo clinic patients) with diagnosis of BPPV by Dix Hallpike test within the time period of our study and these were prospectively evaluated by the same investigator.

Akkuzu et al ${ }^{[21]}$ studied VEMP changes in 25 patients and 17 healthy controls and we didn't have any controls in our study. Hong et al ${ }^{\text {[22] }}$ measured vestibular evoked myogenic potential in 53 patients with BPPV and 84 healthy subjects. Our study included 30 patients. The study by Akkuzu et al ${ }^{[21]}$ had BPPV patients with mean age 52.9 \pm 11.9 years (range 34-77 years). In our study, the mean age group was 50 years. The youngest patient was 32 years and the oldest 68 years. Males and females were found to be almost equal in number with a slight female preponderance.

In the study by Murofushi et al ${ }^{[22]}$, the mean \pm S.D latencies of p13 and $\mathrm{n} 23$ were $11.8 \pm 0.86$ and $20.8 \pm 2.2 \mathrm{~ms}$, respectively. In the study by Akkuzu et al ${ }^{[21]}$ the mean latencies at p13 and n23 in the 17 control subjects were $13.7 \pm 1.0$ and $22.1 \pm 1.9 \mathrm{~ms}$, respectively. In our study, the mean latencies at p13 and $\mathrm{n} 23$ for the $90 \%$ of patients were $12.0 \pm 1.1$ and $20.6 \pm 2.0 \mathrm{~ms}$ respectively which fell in the normal range as given in literature. In the remaining three patients, the mean latencies at p13 were $13 \mathrm{~ms}, 15 \mathrm{~ms}$ and $16 \mathrm{~ms}$ and latencies at n23 were $26 \mathrm{~ms}, 21 \mathrm{~ms}$ $28 \mathrm{~ms}$ which showed increased latencies of $\mathrm{p} 1$ and $\mathrm{n} 1$.

So none of the electrophysiologic tests in isolation seemed to be beneficial for any particular vestibular disorder. We should be judicious and incorporate a standard battery of tests for diagnosis of a peripheral vestibular disorder.

Click stimulus in performing VEMP are the most reproducible, symmetric, and technically easy. Normal values for their thresholds, latencies, amplitudes, and side-to-side differences have been defined. They best fit the role of a screening test of otolith function. The remaining techniques should be used when specifically indicated. When click or tone burst-evoked VEMPs are absent, technical and physiologic causes (inadequate 
stimulus intensity, inadequate SCM activation, conductive hearing loss, and age effects) should first be sought. Audiometry should be performed to determine whether conductive hearing loss is present, and an alternate stimulus that bypasses the middle ear (taps, bone vibration) should be used $^{\text {[24]. }}$.

We performed VEMP using click stimulus. 25\% of patients had conductive hearing loss, two had normal VEMP and one had abnormal VEMP.

Thus, in conclusion BPPV doesn't have any specific changes in VEMP as only 3 of the thirty patients studied, showed any changes. So VEMP does not seem useful as a diagnostic tool in patients with BPPV.

\section{References}

1. Bickford RG, Jacobson JL, Cody DT. Nature of Average Evoked Potentials to Sound and Other Stimuli in Man. Ann N Y Acad Sci. 1964; 112:204-23.

2. Murofushi $T$, Iwasaki $S$, Ushio $M$. Recovery of vestibular evoked myogenic potentials after a vertigo attack due to vestibular neuritis. Acta Otolaryngol 2006; 126:364Y7.

3. Parnes LS, McClure JA. Free-floating endolymph particles: a new operative finding during posterior semicircular canal occlusion. Laryngoscope 1992; 102:988Y92.

4. Akkuzu G, Akkuzu B, Ozluoglu LN. Vestibular evoked myogenic potentials in benign paroxysmal positional vertigo and Meniere's disease. Eur Arch Otorhinolaryngol 2006; 263:510Y7.

5. Gacek RR. Pathology of benign paroxysmal positional vertigo revisited. Ann Otol Rhinol Laryngol 2003; 112:574Y82.

6. Lee KJ, Kim MS, Jin Son E et al. The usefulness of rectified VEMP: Clin Exp Otorhinolaryngol. 2008; 1: 143-147

7. Halmagyi GM, Colebatch JG. Vestibular evoked myogenic potentials in the sternomastoid muscle are not of lateral canal origin. Acta Otolaryngol Suppl.1995; 520:1-3.

8. Isaradisaikul $\mathrm{S}$, Navacharoen $\mathrm{N}$, Hanprasertpong C, Kangsanarak J. Cervical Vestibular-Evoked Myogenic Potentials: Norms and Protocols. Int J Otorhinolaryngol. 2012; 2012:913515.

9. Wuyts FL, Furman J, Vanspauwen R, Van De Heyning P. Vestibular function testing. Curr Opin Neurol. 2007; 20:19-24.

10. Isaradisaikul S, Navacharoen N, Strong DA, Thongyai K, Jenkins HA. Vestibular evoked myogenic potentials. Thai J Otolaryngol, Head and Neck Surgery. 2007; 8:14-20.

11. Zhou G, Cox LC. Vestibular evoked myogenic potentials: history and overview. Am J Audiol. 2004; 13:135-43.

12. Murofushi T, Matsuzaki M, Wu CH. Short tone burst-evoked myogenic potentials on the sternocleidomastoid muscle: are these potentials also of vestibular origin? Arch Otolaryngol Head Neck Surg. 1999;125:660-64.

13. Basta D, Todt I, Ernst A. Normative data for P1/N1-latencies of vestibular evoked myogenic potentials induced by air- or bone-conducted tone bursts. Clin Neurophysiol. 2005; 116:2216-19.

14. Colebatch JG, Halmagyi GM, Skuse NF. Myogenic potentials generated by a click evoked vestibulocollic reflex. J Neurol Neurosurg Psychiatry. 1994; 57:190- 97.

15. Gacek RR. Pathology of benign paroxysmal positional vertigo revisited. Ann Otol Rhinol Laryngol. 2003; 112:57482.

16. Schuknecht HF. Cupulolithiasis. Arch Otolaryngol. 1969; 90:765-78

17. Hall SF, Ruby RR, McClure JA. The mechanics of benign paroxysmal vertigo. $\mathrm{J}$ Otolaryngol. 1979; 8:151-58.

18. Epley JM. Benign paroxysmal vertigo (canalithiasis): diagnosis and non-surgical 
treatment in Dizziness and Balance Disorders, I. K. Arenber, Ed., pp. 545559, Kugler, Amsterdam, The Netherlands, 1993.

19. Epley JM. The Canalith Repositioning Procedure: for treatment of benign paroxysmal positional vertigo. Otolaryngol- Head and Neck Surgery.1992;107(3):399-404.

20. Humphriss RL, Baguley DM, Sparkes V, Peerman SE, Moffat DA. Contraindications to the DixHallpikemanoeuvre: a multidisciplinary review. Int J Audiol. 2003; 42:166-73.

21. Akkuzu G, Akkuzu B, Ozluoglu LN, Vestibular evoked myogenic potentials in benign paroxysmal positional vertigo and Meniere's disease, Eur Arch Otorhinolaryngol 2006; 263: 510-517.

22. Hong S, Park D, Cha C. Vestibular evoked myogenic potentials in patients with benign paroxysmal positional vertigo involving each semicircular canal. Am J Otolaryngol.2008 29: 184-187.

23. Murofushi T1, Shimizu K, Takegoshi H, Cheng PW. Diagnostic value of prolonged latencies in the vestibular evoked myogenic potential. Arch Otolaryngol Head Neck Surg. 2001; 127:1069-72.

24. Welgampola MS, Colebatch JG, Characteristics and clinical applications of vestibular-evoked myogenic potentials. Neurology 2005; 64:1682-88. 